\title{
Mixed-Timescale Beamforming and Power Splitting for Massive MIMO Aided SWIPT IoT Network
}

\author{
Xihan Chen ${ }^{1}$, Hei Victor Cheng ${ }^{2}$, An Liu ${ }^{1}$, Kaiming Shen ${ }^{2}$, and Min-Jian Zhao ${ }^{1}$
}

\begin{abstract}
Traditional simultaneous wireless information and power transfer (SWIPT) with power splitting assumes perfect channel state information (CSI), which is difficult to obtain especially in the massive multiple-input-multiple-output (MIMO) regime. In this letter, we consider a mixed-timescale joint beamforming and power splitting (MJBP) scheme to maximize general utility functions under a power constraint in the downlink of a massive MIMO SWIPT IoT network. In this scheme, the transmit digital beamformer is adapted to the imperfect CSI, while the receive power splitters are adapted to the long-term channel statistics only due to the consideration of hardware limit and signaling overhead. The formulated optimization problem is solved using a mixed-timescale online stochastic successive convex approximation (MO-SSCA) algorithm. Simulation results reveal significant gain over the baselines.
\end{abstract}

Index Terms-SWIPT, massive MIMO, mixed-timescale joint beamforming and power splitting, online stochastic successive convex approximation.

\section{INTRODUCTION}

The Internet of Things (IoT) [1] is a revolutionary communication paradigm to provide massive connectivity for the next-generation wireless cellular networks. The limited battery life of devices poses a significant challenge for designing green and sustainable IoT. One promising solution is to leverage the simultaneous wireless information and power transfer (SWIPT) with radio frequency to prolong the IoT network, due to its ability to provide cost-effective and perpetual power source [2]. This requires receiver circuits to decode information and harvest energy from the same received signal independently and simultaneously, which renders SWIPT impractical.

To overcome these limitations, the telecommunication industry is increasingly turning towards power splitting (PS), a receiver architecture that divides the received signal into two streams of different power for decoding information and harvesting energy. Based on the PS architecture, [3] considers a multiuser joint beamforming and power splitting design problem under QoS constraints and proposes a semidefinite relaxation-based algorithm. To further reduce the computational complexity, an second-order cone programming relax-

This work was supported by the Science and Technology Program of Shenzhen, China, under Grant JCYJ20170818113908577, and the National Natural Science Foundation of China under Project No. 61571383. The work of An Liu was supported by the China Recruitment Program of Global Young Experts.

Xihan Chen, An Liu and Min-Jian Zhao are with the College of Information Science and Electronic Engineering, Zhejiang University, Hangzhou 310027, China (e-mail: chenxihan@zju.edu.cn, anliu@zju.edu.cn, mjzhao@zju.edu.cn).

Hei Victor Cheng, and Kaiming Shen are with the Electrical and Computer Engineering Department, University of Toronto, Toronto, ON M5S 3G4, Canada (e-mail: hei.cheng@utoronto.ca, kshen@ece.utoronto.ca). ation method is proposed in [4]. Recently, [5] combines the SWIPT and massive multiple-input-multiple-output (MIMO) to further improve the spectral and energy efficiency of IoT networks. The aforementioned works focus on optimizing the weighted sum of objective function under perfect CSI, which is difficult to obtain in the massive MIMO regime due to the large number of antennas and the limited pilot sequences [6]. In such scenarios, it is more reasonable to consider a mixed-timescale optimization of the long-term performance of the network, which only requires imperfect CSI plus the knowledge of channel statistics [7]. To the best of our knowledge, this is first work on mixed-timescale optimization for massive MIMO aided SWIPT IoT network.

Contribution of this letter includes the algorithm design for mixed-timescale joint beamforming and power splitting (MJBP) scheme for the downlink transmission of massive MIMO aided SWIPT IoT network, to maximize a general network utility. Specifically, the digital beamformer is adapted to the imperfect CSI, while the power spiltters are adapted to the long-term channel statistics due to the consideration of hardware limit and signaling overhead. We propose a mixedtimescale online stochastic successive convex approximation (MO-SSCA) algorithm to solve this joint optimization problem. Simulations verify the advantages of the proposed MJBP scheme over the baselines.

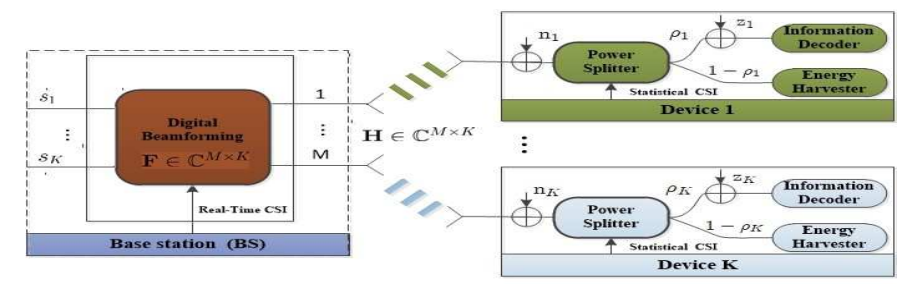

Fig. 1: Architecture of the proposed MJBP scheme.

\section{System Model AND Problem Formulation}

Consider the downlink of a massive MIMO aided SWIPT IoT network, where the base station (BS) is equipped with $M$ antennas to simultaneously serve $K$ single-antenna IoT devices. As illustrated in Fig. 1, the BS employs digital beamformer $\mathbf{F} \triangleq\left[\boldsymbol{f}_{1}, \cdots, \boldsymbol{f}_{K}\right] \in \mathbb{C}^{M \times K}$ to spatially multiplex devices and manage the multi-device interference, while device $k$ applies the power splitter $\rho_{k}\left(0 \leq \rho_{k} \leq 1\right)$ to coordinate information decoding and energy harvesting from the received signal. With MJBP, both the digital beamformer and the power splitters are optimized at the BS. Furthermore, the digital beamformer is adapted to instantaneous CSI. For 
the power splitter implemented at each device, it is adapted to long-term channel statistics due to following reasons: 1) the hardware capability of the IoT device is limited, and thus the power splitter cannot be changed frequently due to hardware limitations [1]; 2) such design can reduce the signaling overhead of sending $\rho_{k}, \forall k$, to the corresponding device, especially when the number of devices is large.

We consider flat fading channels with block fading model, but the proposed algorithm can be easily modified to cover the frequency selective channels. The channel $\mathbf{H} \triangleq$ $\left[\boldsymbol{h}_{1}, \cdots, \boldsymbol{h}_{K}\right] \in \mathbb{C}^{M \times K}$ is assumed to be constant within each block of length $T$. In this case, the received signal splitted to the information decoder (ID) of device $k$ is given by $y_{k}^{I}=$ $\sqrt{\rho_{k}}\left(\boldsymbol{h}_{k}^{H} \sum_{m=1}^{K} \boldsymbol{f}_{m} s_{m}+n_{k}\right)+z_{k}$, where $s_{m} \sim \mathcal{C N}(0,1)$ is the data symbol for device $m, n_{k} \sim \mathcal{C N}\left(0, \sigma_{k}^{2}\right)$ is the additive noise (AN) at the PS of device $k$, and $z_{k} \sim \mathcal{C N}\left(0, \delta_{k}^{2}\right)$ is the AN introduced by the ID at device $k$. Meanwhile, the received signal splitted to the energy harvester $(\mathrm{EH})$ is given by $y_{k}^{E}=\sqrt{1-\rho_{k}}\left(\boldsymbol{h}_{k}^{H} \sum_{m=1}^{K} \boldsymbol{f}_{m} s_{m}+n_{k}\right)$.

An achievable ergodic rate [6] at device $k$ is given by

$$
\begin{aligned}
\hat{r}_{k}^{\circ}\left(\rho_{k}, \mathbf{F}\right) & =\mathbb{E}_{\mathbf{H}}\left[\log _{2}\left(1+\Gamma_{k}\left(\rho_{k}, \mathbf{F}, \mathbf{H}\right)\right)\right] \\
& -\frac{1}{T} \sum_{m=1}^{K} \log _{2}\left(1+\frac{T}{\rho_{k} \sigma_{k}^{2}+\delta_{k}} \operatorname{Var}\left(\boldsymbol{h}_{k}^{H} \boldsymbol{f}_{m}\right),\right.
\end{aligned}
$$

where $\Gamma_{k}\left(\rho_{k}, \mathbf{F}, \mathbf{H}\right)$ is the SINR of device $k$ with

$$
\Gamma_{k}\left(\rho_{k}, \mathbf{F}, \mathbf{H}\right)=\frac{\rho_{k}\left|\boldsymbol{h}_{k}^{H} \boldsymbol{f}_{k}\right|^{2}}{\rho_{k}\left(\sum_{m \neq k}^{K}\left|\boldsymbol{h}_{k}^{H} \boldsymbol{f}_{m}\right|^{2}+\sigma_{k}^{2}\right)+\delta_{k}^{2}} .
$$

In practice, perfect CSI is challenging to obtain due to device mobility, processing latency and other limitations. Thus, we model the channel imperfection as $\boldsymbol{h}_{k}=\hat{\boldsymbol{h}}_{k}+\boldsymbol{\phi}_{k}$, where $\hat{\boldsymbol{h}}_{k}$ is the estimated channel from from BS to device $k$, $\phi_{k} \sim \mathcal{C N}\left(0, \omega_{k}^{2} \mathbf{I}_{M}\right)$ is the channel error independent of $\hat{\boldsymbol{h}}_{k}$, and $\omega_{k}^{2}$ is the variance of the channel error. Consequently, the achievable rate is obtained by replacing $\boldsymbol{h}_{k}$ in (1) with $\hat{\boldsymbol{h}}_{k}+\phi_{k}$. For convenience, we let $\hat{\mathbf{H}} \triangleq\left[\hat{\boldsymbol{h}}_{1}, \cdots, \hat{\boldsymbol{h}}_{K}\right]$, and $\phi \triangleq\left[\boldsymbol{\phi}_{1}^{T}, \cdots, \boldsymbol{\phi}_{K}^{T}\right]^{T}$. Further, we define $\Theta \triangleq\{\mathbf{F}(\hat{\mathbf{H}}) \in$ $\Lambda, \forall \hat{\mathbf{H}}\}$ as the collection of short-term optimization variables for all possible estimated channel states $\hat{\mathbf{H}}$, where $\Lambda \triangleq$ $\left\{\mathbf{F} \mid \operatorname{Tr}\left(\mathbf{F F} \mathbf{F}^{H}\right) \leq P_{\max }\right\}$ is the feasible set of $\mathbf{F}$.

Proposition 1 : The ergodic rate at device is $k$ bounded as $\hat{r}_{k}^{\circ}\left(\rho_{k}, \boldsymbol{\Theta}\right) \leq \bar{r}_{k}\left(\rho_{k}, \boldsymbol{\Theta}\right) \triangleq \mathbb{E}_{\hat{\mathbf{H}}, \phi_{k}}\left[\log _{2}(1+\right.$ $\left.\left.\Gamma_{k}\left(\rho_{k}, \mathbf{F}, \hat{\boldsymbol{h}}_{k}, \boldsymbol{\phi}_{k}\right)\right)\right]$, and $\hat{r}_{k}^{\circ}\left(\rho_{k}, \boldsymbol{\Theta}\right) \geq$

$$
\bar{r}_{k}\left(\rho_{k}, \boldsymbol{\Theta}\right)-\frac{1}{T} \sum_{m=1}^{K} \log _{2}\left(1+\frac{T P_{\max }}{\delta_{k}^{2}} \mathbb{E}_{\hat{\mathbf{H}}, \phi_{k}}\left[\left\|\hat{\boldsymbol{h}}_{k}+\boldsymbol{\phi}_{k}\right\|^{2}\right]\right) .
$$

Here the lower bound follows from the properties of variance and the Cauchy-Schwarz inequality. From Proposition 1, optimizing the lower and upper bound provide the same optimal solution. Moreover, as verified in Fig. 2, we find that both bounds are tight. Therefore, we optimize the lower (upper) bound of the ergodic rate at each device as it is more tractable for optimization.

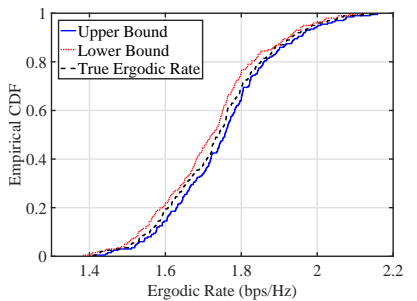

Fig. 2: Cumulative distribution function (CDF) of the lower and upper bound of ergodic rate at each device. The detailed setup is given in Section IV.

The average harvested power conditioned on imperfect CSI $\hat{\boldsymbol{h}}_{k}$ of device $k$ follows a non-linear function [8] and can be expressed as $\bar{e}_{k}^{\circ}\left(\rho_{k}, \mathbf{F} \mid \hat{\boldsymbol{h}}_{k}\right)=\mathbb{E}_{\boldsymbol{\phi}_{k}}\left[\hat{e}_{k}^{\circ}\left(\rho_{k}, \mathbf{F} \mid \hat{\boldsymbol{h}}_{k}, \boldsymbol{\phi}_{k}\right)\right]$, where

$$
\hat{e}_{k}^{\circ}\left(\rho_{k}, \mathbf{F} \mid \hat{\boldsymbol{h}}_{k}, \boldsymbol{\phi}_{k}\right)=\left(\Psi_{k}-S_{k} \Omega_{k}\right) /\left(1-\Omega_{k}\right),
$$

where $S_{k}$ is a constant denoting the maximum harvested power at the $k$ th device, $\Psi_{k} \triangleq \frac{S_{k}}{1+\exp \left(-a_{k}\left(P_{k}-b_{k}\right)\right)}, \Omega_{k} \triangleq$ $\frac{1}{1+\exp \left(a_{k} b_{k}\right)}$, and parameter $a_{k}$ and $b_{k}$ are constants related to the circuit specifications, and $P_{k} \triangleq\left(1-\rho_{k}\right)\left(\sum_{m=1}^{K} \mid\left(\hat{\boldsymbol{h}}_{k}+\right.\right.$ $\left.\left.\phi_{k}\right)\left.^{H} \boldsymbol{f}_{m}\right|^{2}+\sigma_{k}^{2}\right)$ is the input RF power for the $k$ th device. Then, the average harvested power of user $k$ is defined as $\bar{e}_{k}\left(\rho_{k}, \boldsymbol{\Theta}\right)=\mathbb{E}_{\hat{\mathbf{H}}}\left[\bar{e}_{k}^{\circ}\left(\rho_{k}, \mathbf{F}(\hat{\mathbf{H}}) \mid \hat{\boldsymbol{h}}_{k}\right)\right]$.

We are interested in a mixed-timescale joint optimization of digital beamformer and power splitter to balance the average ergodic rate and the average harvested power. This can be formulated as the following network utility maximization problem:

$$
\mathcal{P}: \max _{\boldsymbol{\rho} \in \Phi, \Theta} \sum_{k=1}^{K} g\left(\bar{\eta}_{k}\left(\rho_{k}, \mathbf{\Theta}\right)\right),
$$

where $\bar{\eta}_{k}\left(\rho_{k}, \boldsymbol{\Theta}\right) \triangleq \bar{r}_{k}\left(\rho_{k}, \boldsymbol{\Theta}\right)+\gamma_{k} \bar{e}_{k}\left(\rho_{k}, \boldsymbol{\Theta}\right)$ with the corresponding weight $\gamma_{k}$ is a weighted sum of the average ergodic rate and the harvested power, $\Phi \triangleq\left\{\boldsymbol{\rho}=\left[\rho_{1}, \cdots, \rho_{K}\right]^{T} \mid \rho_{k} \in\right.$ $(0,1], \forall k\}$ is the feasible set of power splitters. The utility function $g\left(\bar{\eta}_{k}\right)$ is a continuously differentiable and concave function of $\bar{\eta}_{k}$. Moreover, $g\left(\bar{\eta}_{k}\right)$ is non-decreasing w.r.t. $\bar{\eta}_{k}$, and its derivative $\nabla_{\bar{\eta}_{k}} g\left(\bar{\eta}_{k}\right)$ is Lipschitz continuous.

\section{Online OptimizATION AlgORithm}

In this section, we propose a MO-SSCA algorithm to solve the mixed-timescale stochastic non-convex optimization problem $\mathcal{P}$, and summarize it in Algorithm 1. In MO-SSCA, we focus on a coherence time of channel statistics, where the time is divided into $T_{f}$ frames and each frame consists of $T_{s}$ time slots. At beginning, the BS initializes the MO-SSCA algorithm with power splitter $\boldsymbol{\rho}^{0}$ and a weight vector $\boldsymbol{v}^{0}$. In subsequent, $\rho$ and $v$ are updated once at the end of each frame. Then we elaborate the implementation details of the iteration of the MO-SSCA algorithm at the $t$-th frame.

\section{A. Short-term FP-BCD Algorithm}

At time slot $i \in \mathcal{T}_{t} \triangleq\left[t T_{s}+1,(t+1) T_{s}\right]$ within the $t$ th frame, BS obtains the estimated channel $\hat{\mathbf{H}}(i)$ by uplink channel training. Based upon the current $\boldsymbol{\rho}^{t}, \boldsymbol{v}^{t}$, and $\hat{\mathbf{H}}(i)$, we 


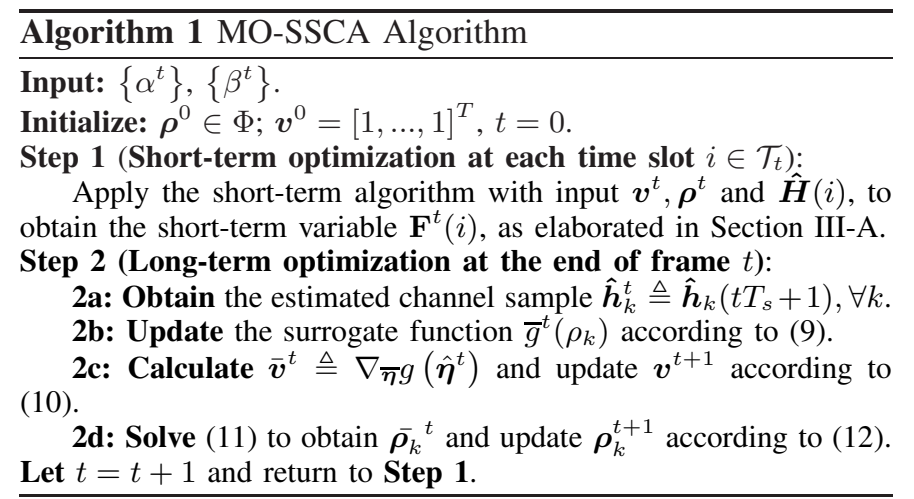

can obtain digital beamforming by maximizing the a weighted sum of the average data rate and the average harvested power conditioned on imperfect CSI, which can be formulated as

$$
\mathcal{P}_{2}(\boldsymbol{\rho}, \boldsymbol{v}, \hat{\mathbf{H}}): \max _{\mathbf{F} \in \Lambda} \sum_{k=1}^{K} v_{k} \bar{\eta}_{k}^{\circ}\left(\rho_{k}, \mathbf{F} \mid \hat{\boldsymbol{h}}_{k}\right)
$$

where $\bar{\eta}_{k}^{\circ}\left(\rho_{k}, \mathbf{F} \mid \hat{\boldsymbol{h}}_{k}\right) \triangleq \bar{r}_{k}^{\circ}\left(\rho_{k}, \mathbf{F} \mid \hat{\boldsymbol{h}}_{k}\right)+\gamma_{k} \bar{e}_{k}^{\circ}\left(\rho_{k}, \mathbf{F} \mid \hat{\boldsymbol{h}}_{k}\right)$ with $\bar{r}_{k}^{\circ}\left(\rho_{k}, \mathbf{F} \mid \hat{\boldsymbol{h}}_{k}\right)=\mathbb{E}_{\boldsymbol{\phi}}\left[\hat{r}_{k}^{\circ}\left(\rho_{k}, \mathbf{F} \mid \hat{\boldsymbol{h}}_{k}, \boldsymbol{\phi}_{k}\right)\right] \quad$ and $\hat{r}_{k}^{\circ}\left(\rho_{k}, \mathbf{F} \mid \hat{\boldsymbol{h}}_{k}, \boldsymbol{\phi}_{k}\right)=\log _{2}\left(1+\Gamma_{k}\left(\rho_{k}, \mathbf{F}, \hat{\boldsymbol{h}}_{k}, \boldsymbol{\phi}_{k}\right)\right)$, and $\mathcal{P}_{2}\left(\boldsymbol{\rho}^{t}, \boldsymbol{v}^{t}, \hat{\mathbf{H}}(i)\right)$ is solved at time slot $i \in \mathcal{T}_{t}$.

Since the objective function $\bar{\eta}_{k}^{\circ}\left(\rho_{k}, \mathbf{F} \mid \hat{\boldsymbol{h}}_{k}\right)$ contains expectation operators, it does not have a closed-form expression. To address the challenge, we resort to the Sample Average Approximation (SAA) method [9]. Specifically, a total of $N$ samples are generated for $\phi_{k}$ independently drawn from the distribution $\mathcal{C N}\left(0, \omega_{k}^{2} \mathbf{I}_{M}\right)$, and the $n$-th sample of $\phi_{k}$ is defined as $\phi_{k}^{n}$. In this case, the SAA version of $\mathcal{P}_{2}(\boldsymbol{\rho}, \boldsymbol{v}, \hat{\mathbf{H}})$ is formulated as $\mathcal{P}_{3}(\boldsymbol{\rho}, \boldsymbol{v}, \hat{\mathbf{H}})$ : $\max _{\mathbf{F} \in \Lambda} \quad \frac{1}{N} \sum_{k=1}^{K} \sum_{n=1}^{N} v_{k} \hat{\eta}_{k}^{\circ}\left(\rho_{k}, \mathbf{F} \mid \hat{\boldsymbol{h}}_{k}, \boldsymbol{\phi}_{k}^{n}\right), \quad$ where $\hat{\eta}_{k}^{\circ}\left(\rho_{k}, \mathbf{F} \mid \hat{\boldsymbol{h}}_{k}, \boldsymbol{\phi}_{k}^{n}\right)=\hat{r}_{k}^{\circ}\left(\rho_{k}, \mathbf{F} \mid \hat{\boldsymbol{h}}_{k}, \boldsymbol{\phi}_{k}^{n}\right)+\gamma_{k} \hat{e}_{k}^{\circ}\left(\rho_{k}, \mathbf{F} \mid \hat{\boldsymbol{h}}_{k}, \boldsymbol{\phi}_{k}^{n}\right)$.

However, solving problem $\mathcal{P}_{3}(\boldsymbol{\rho}, \boldsymbol{v}, \hat{\mathbf{H}})$ is still challenging due to the nonlinear fractional term in $\hat{r}_{k}$ and coupling in the power constraint. To this end, we apply the Lagrangian dual transform method [10] to recast problem $\mathcal{P}_{3}(\boldsymbol{\rho}, \boldsymbol{v}, \hat{\mathbf{H}})$ into a more tractable yet equivalent form, using the following lemma.

Lemma 1. The optimal digital beamforming $\mathbf{F}^{*}$ solves the problem in (1) if and only if it solves

$$
\max _{\mathbf{F} \in \Lambda} \frac{1}{N} \sum_{n=1}^{N} \sum_{k=1}^{K} f\left(\mathbf{F}, q_{k}^{n}, \phi_{k}^{n}\right)
$$

where $f\left(\mathbf{F}, q_{k}^{n}, \boldsymbol{\phi}_{k}^{n}\right) \triangleq v_{k}\left(\log _{2}\left(1+q_{k}^{n}\right)-q_{k}^{n}+\left(\rho_{k}\left(\Gamma_{k}^{n}+\right.\right.\right.$ $\left.\left.\left.\sigma_{k}^{2}\right)+\delta_{k}^{2}\right)^{-1}\left(1+q_{k}^{n}\right) \rho_{k}\left|\tilde{\boldsymbol{h}}_{k, n}^{H} \boldsymbol{f}_{k}\right|^{2}+\gamma_{k} \hat{e}_{k}^{\circ}\left(\mathbf{F}, q_{k}^{n}, \boldsymbol{\phi}_{k}^{n}, w_{k}^{n}\right)\right)$, $\Gamma_{k}^{n} \triangleq \sum_{m=1}^{K}\left|\tilde{\boldsymbol{h}}_{k, n}^{H} \boldsymbol{f}_{m}\right|^{2}$ with $\tilde{\boldsymbol{h}}_{k, n} \triangleq \boldsymbol{\phi}_{k}^{n}+\hat{\boldsymbol{h}}_{k}$, and $q_{k}^{n} \triangleq$ $\frac{\rho_{k}\left|\tilde{\boldsymbol{h}}_{k, n}^{H} \boldsymbol{f}_{k}\right|^{2}}{\rho_{k}\left(\sum_{m \neq k}^{K}\left|\tilde{\boldsymbol{h}}_{k, n}^{H} \boldsymbol{f}_{m}\right|^{2}+\sigma_{k}^{2}\right)+\delta_{k}^{2}}$ is the optimal auxiliary variable introduced for each ratio term.

In subsequent, we use the complex quadratic transformation
[10] to equivalently recast problem (3) as

$$
\max _{\mathbf{F} \in \Lambda, \boldsymbol{w}, \boldsymbol{q}} \frac{1}{N} \sum_{n=1}^{N} \sum_{k=1}^{K} \hat{r}_{k}\left(\mathbf{F}, q_{k}^{n}, \boldsymbol{\phi}_{k}^{n}, w_{k}^{n}\right)+\hat{e}_{k}^{\circ}\left(\mathbf{F}, q_{k}^{n}, \boldsymbol{\phi}_{k}^{n}, w_{k}^{n}\right)
$$

where

$$
\begin{aligned}
& \hat{r}_{k}\left(\mathbf{F}, q_{k}^{n}, \boldsymbol{\phi}_{k}^{n}, w_{k}^{n}\right) \triangleq \sqrt{v_{k} \rho_{k}\left(1+q_{k}^{n}\right)} \operatorname{Re}\left\{\boldsymbol{f}_{k}^{H} \tilde{\boldsymbol{h}}_{k, n}\left(w_{k}^{n}\right)^{H}\right\} \\
& +\left(w_{k}^{n}\right)^{H} w_{k}^{n}\left(\rho_{k}\left(\Gamma_{k}^{n}+\sigma_{k}^{2}\right)+\delta_{k}^{2}\right)-q_{k}^{n}+\log _{2}\left(1+q_{k}^{n}\right) .
\end{aligned}
$$

$\boldsymbol{q}=\left[\boldsymbol{q}_{1}^{T}, \cdots, \boldsymbol{q}_{K}^{T}\right]^{T}$ with $\boldsymbol{q}_{k}=\left[q_{k}^{1}, \cdots, q_{k}^{N}\right]^{T}$, and $\boldsymbol{w}=$ $\left[\boldsymbol{w}_{1}^{T}, \cdots, \boldsymbol{w}_{K}^{T}\right]^{T}$ with $\boldsymbol{w}_{k}=\left[w_{k}^{1}, \cdots, w_{k}^{N}\right]^{T}$ is the auxiliary variable vector. Observing that the constraints are separable with respect to the three blocks of variables, i.e., $\boldsymbol{q}, \boldsymbol{w}$, and $\mathbf{F}$, we shall focus on designing a fractional programming block coordinate descent (FP-BCD) algorithm to find a stationary point of problem (4), and summarize it in Algorithm 2. For problem (4), this amounts to the following steps:

$\overline{\text { Algorithm } 2 \text { Short-term FP-BCD Algorithm for problem (4) }}$

Input: $\boldsymbol{v}, \boldsymbol{\rho}, \hat{\mathbf{H}}$, the sample number $N$.

Initialization: Initialize $\mathbf{F}$ to feasible values.

\section{Repeat}

Step 1 : Update $q$ according to (5).

Step 2 : Update $\boldsymbol{w}$ according to (6).

Step 3 : Update $\mathbf{F}$ by solving problem (8) using CVX.

until the value of objective function in (4) converges

1) Optimization of $\boldsymbol{q}$ : The optimal $\boldsymbol{q}^{*}$ is given by

$$
\left(q_{k}^{n}\right)^{*}=\frac{\rho_{k}\left|\tilde{\boldsymbol{h}}_{k, n}^{H} \boldsymbol{f}_{k}\right|^{2}}{\rho_{k}\left(\Gamma_{k}^{n}-\left|\tilde{\boldsymbol{h}}_{k, n}^{H} \boldsymbol{f}_{k}\right|^{2}+\sigma_{k}^{2}\right)+\delta_{k}^{2}} .
$$

2) Optimization of $\boldsymbol{w}$ : By applying the first-order optimal condition, the optimal $\boldsymbol{w}^{*}$ admits a closed-form solution as:

$$
\left(w_{k}^{n}\right)^{*}=\left(\rho_{k}\left(\Gamma_{k}^{n}+\sigma_{k}^{2}\right)+\delta_{k}^{2}\right) \sqrt[-1]{\rho_{k} v_{k}\left(1+q_{k}^{n}\right)} \tilde{\boldsymbol{h}}_{k, n}^{H} \boldsymbol{f}_{k}
$$

3) Optimization of $\mathbf{F}$ : The subproblem w.r.t. $\mathbf{F}$ is nonconvex due to the involvement of the non-linear energy harvesting model. To overcome this difficulty, we first transform it into a more tractable yet equivalent form by the introduction of new auxiliary variables $0 \leq \varsigma_{k}^{n} \leq \hat{e}_{k}^{\circ}\left(\mathbf{F}, q_{k}^{n}, \phi_{k}^{n}, w_{k}^{n}\right)$ and some manipulations, which can be expressed as

$$
\begin{aligned}
& \mathcal{P}_{4}: \max _{\mathbf{F} \in \Lambda, \alpha_{k}^{n} \geq 0} \frac{1}{N} \sum_{n=1}^{N} \sum_{k=1}^{K} \hat{r}_{k}\left(\mathbf{F}, q_{k}^{n}, \boldsymbol{\phi}_{k}^{n}, w_{k}^{n}\right)+\varsigma_{k}^{n} \\
& \text { s.t. } \ln \left(1 /\left(\alpha_{k}^{n}+\Omega_{k}^{n}\right)-1\right)+d_{k} \sum_{m=1}^{K}\left|\left(\hat{\boldsymbol{h}}_{k}+\boldsymbol{\phi}_{k}^{n}\right)^{H} \boldsymbol{f}_{m}\right|^{2}+c_{k} \geq 0,
\end{aligned}
$$

where $d_{k} \triangleq a_{k}\left(1-\rho_{k}\right)$, and $c_{k} \triangleq a_{k} \sigma_{k}^{2}\left(1-\rho_{k}\right)-a_{k} b_{k}$. Note that the constraint in problem $\mathcal{P}_{4}$ is nonconvex. Thus, we apply the the majorization minimization (MM) method [11] 
to approximate this nonconvex constraint using its first-order Taylor expansion as

$$
\begin{aligned}
& \max _{\mathbf{F} \in \Lambda, \alpha_{k}^{n} \geq 0} \frac{1}{N} \sum_{n=1}^{N} \sum_{k=1}^{K} \hat{r}_{k}\left(\mathbf{F}, q_{k}^{n}, \boldsymbol{\phi}_{k}^{n}, w_{k}^{n}\right)+\varsigma_{k}^{n} \\
& \text { s.t. } d_{k} \sum_{m=1}^{K}\left(\hat{\boldsymbol{h}}_{k}+\boldsymbol{\phi}_{k}^{n}\right)^{H}\left(\tilde{\boldsymbol{f}}_{m} \tilde{\boldsymbol{f}}_{m}^{H}+\tilde{\boldsymbol{f}}_{m} \overline{\boldsymbol{f}}_{m}^{H}+\overline{\boldsymbol{f}}_{m} \tilde{\boldsymbol{f}}_{m}^{H}\right)\left(\hat{\boldsymbol{h}}_{k}+\boldsymbol{\phi}_{k}^{n}\right) \\
& +\ln \left(1 /\left(\tilde{\varsigma}_{k}^{n}+\Omega_{k}^{n}\right)-1\right)+\frac{\varsigma_{k}^{n}-\tilde{\varsigma}_{k}^{n}}{\left(\tilde{\varsigma}_{k}^{n}+\Omega_{k}^{n}-1\right)\left(\tilde{\varsigma}_{k}^{n}+\Omega_{k}^{n}\right)}+c_{k} \geq 0,
\end{aligned}
$$

where $\tilde{\varsigma}_{k}^{n}$ and $\tilde{\boldsymbol{f}_{m}}$ represents the last iteration of $\varsigma_{k}^{n}$ and $\boldsymbol{f}_{m}$, and $\overline{\boldsymbol{f}}_{m} \triangleq \boldsymbol{f}_{m}-\tilde{\boldsymbol{f}}_{m}$. Note that problem (8) is convex, which can be efficiently solved by the CVX toolbox [12].

\section{B. Long-term Optimization}

Before the end of $t$-th frame, device $k$ obtains a full channel sample $\hat{\boldsymbol{h}}_{k}^{t} \triangleq \hat{\boldsymbol{h}}_{k}\left(t T_{s}+1\right)$ and channel error sample $\phi_{k}^{t}$. Based on $\hat{\boldsymbol{h}}_{k}^{t}, \boldsymbol{\phi}_{k}^{t}$ and $\mathbf{F}^{t}(i), \forall i \in \mathcal{T}_{t}$, we preserve the partial concavity of the original function and add the proximal regularization, to construct the concave surrogate function $\bar{g}^{t}\left(\rho_{k}\right)$, resulting in the following

$$
\bar{g}^{t}\left(\rho_{k}\right)=g\left(\tilde{\eta}_{k}^{t}\right)+\left(u_{k}^{t}\right)^{T}\left(\rho_{k}-\rho_{k}^{t}\right)-\tau\left|\rho_{k}-\rho_{k}^{t}\right|^{2},
$$

where $\tau>0$ is a postive constant; the recursive approximation of the weighted sum of the data rate and the harvested power $\tilde{\eta}_{k}$ is given by

$$
\tilde{\eta}_{k}^{t}=\left(1-\alpha_{t}\right) \tilde{\eta}_{k}^{t-1}+\frac{\alpha_{t}}{N} \sum_{n=1}^{N} \sum_{i \in \mathcal{T}_{t}} \frac{\hat{\eta}_{k}^{\circ}\left(\rho_{k}^{t}, \mathbf{F}^{t}(i) \mid \hat{\boldsymbol{h}}_{k}^{i}, \boldsymbol{\phi}_{k}^{n}(i)\right)}{\left|\mathcal{T}_{t}\right|},
$$

with $\tilde{\eta}_{k}^{-1}=0$, and $\alpha_{t} \in(0,1]$ is a step-sizes sequence to be properly chosen; the recursive approximation of the partial derivative $\nabla_{\rho_{k}} g\left(\tilde{\eta}_{k}\right)$ is given by

$$
u_{k}^{t}=\left(1-\alpha_{t}\right) u_{k}^{t-1}+\alpha_{t} J_{\rho_{k}}\left(\rho_{k}^{t}, \mathbf{F}^{t}(i) \mid \hat{\boldsymbol{h}}_{k}^{t}, \boldsymbol{\phi}_{k}^{t}\right) \nabla_{\bar{\eta}_{k}} g\left(\tilde{\eta}_{k}^{t}\right),
$$

with $u_{k}^{-1}=0, J_{\rho_{k}}\left(\rho_{k}^{t}, \mathbf{F}^{t}(i) \mid \hat{\boldsymbol{h}}_{k}^{t}, \boldsymbol{\phi}_{k}^{t}\right)$ is the gradient of $\hat{\eta}_{k}^{\circ}\left(\rho_{k}^{t}, \mathbf{F}^{t}(i) \mid \hat{\boldsymbol{h}}_{k}^{t}, \boldsymbol{\phi}_{k}^{t}\right)$ w.r.t. $\rho_{k}$ at $\rho_{k}=\rho_{k}^{t}$ and $\mathbf{F}=\mathbf{F}^{t}(i)$. Moreover, the weight vector $\boldsymbol{v}$ is updated as

$$
v_{k}^{t+1}=\left(1-\beta_{t}\right) v_{k}^{t}+\beta_{t} \bar{v}_{k}^{t},
$$

with $v_{k}^{t}=\nabla_{\bar{\eta}} g_{k}\left(\tilde{\eta}_{k}^{t}\right)$, where $\beta_{t} \in(0,1]$ is a step-sizes sequence satisfying $\sum_{t} \beta_{t}=\infty, \sum_{t}\left(\beta_{t}\right)^{2}<\infty$. Moreover, the optimal power splitting ratio for device $k$ can be obtained by solving the following quadratic optimization problem, i.e.,

$$
\max _{\rho_{k} \in \Phi} \bar{g}^{t}\left(\rho_{k}\right) .
$$

By applying the first-order optimality condition, it yields the closed-form solution $\bar{\rho}_{k}^{t}=\mathbb{P}_{\Phi}\left[\rho_{k}^{t}+\frac{u_{k}^{t}}{2 \tau}\right]$, where $\mathbb{P}_{\Phi}[\cdot]$ denotes the projection onto the feasible region $\Phi$. Consequently, the long-term variable $\rho_{k}$ is updated as

$$
\rho_{k}^{t+1}=\left(1-\beta_{t}\right) \rho_{k}^{t}+\beta_{t} \bar{\rho}_{k}^{t} .
$$

Remark 1 : Note that the stationary weight vector $v_{k}^{*}=$ $\nabla_{\bar{\eta}_{k}} g_{k}\left(\tilde{\eta}_{k}^{*}\right)$ has captured the nature of the utility function. However, it is difficult to obtain $v^{*}$, since it in turn depends on the stationary solution $\rho^{*}$. Therefore, the basic idea of the proposed algorithm is to iteratively update the long-term variable $\boldsymbol{\rho}^{t}$ and the weight vector $\boldsymbol{v}^{t}$ such that $\boldsymbol{\rho}^{t}$ and $\boldsymbol{v}^{t}$ converge to a stationary solution $\rho^{*}$ and the corresponding stationary weight vector $v^{*}$, respectively.

\section{Convergence Analysis}

The following theorem states that Algorithm 2 converges to a stationary point of $\mathcal{P}_{2}(\boldsymbol{\rho}, \boldsymbol{v}, \hat{\mathbf{H}})$ up to certain convergence error which vanishes to zero exponentially as $N \rightarrow \infty$.

Theorem 2 (Convergence of Algorithm 2). Suppose problem $\mathcal{P}_{2}(\boldsymbol{\rho}, \boldsymbol{v}, \hat{\mathbf{H}})$ has a discrete set of stationary points, denoted by $\mathcal{F}^{*}(\boldsymbol{\rho}, \boldsymbol{v}, \hat{\mathbf{H}})$. Let $\mathbf{F}^{N}(\boldsymbol{\rho}, \boldsymbol{v}, \hat{\mathbf{H}})$ denote the limiting point of the sequence generated by Algorithm 2 with input parameter $\boldsymbol{\rho}, \boldsymbol{v}, \hat{\mathbf{H}}$ and sample number $N$. Then for every small positive number $\epsilon>0$, there exist positive constants $\hat{a}(\epsilon)$ and $\hat{b}(\epsilon)$, independent of $N$, such that

$\operatorname{Pr}\left\{\min _{\mathbf{F} \in \mathcal{F}^{*}(\boldsymbol{\rho}, \boldsymbol{v}, \hat{\mathbf{H}})}\left\|\mathbf{F}^{N}(\boldsymbol{\rho}, \boldsymbol{v}, \hat{\mathbf{H}})-\mathbf{F}(\boldsymbol{\rho}, \boldsymbol{v}, \hat{\mathbf{H}})\right\| \geq \epsilon\right\} \leq p(\epsilon, N)$, for $N$ sufficiently large, where $p(\epsilon, N) \triangleq \hat{a}(\epsilon) e^{-N \hat{b}(\epsilon)}$.

Proof: Specifically, the proposed FP-BCD algorithm falls in the MM framework and similar proof is provided in [13]. From Theorem 4.4 in [11], every limiting point $\mathbf{F}^{N}$ of sequence generated by the short-term FP-BCD algorithm is a stationary point of problem $\mathcal{P}_{3}(\boldsymbol{\rho}, \boldsymbol{v}, \hat{\mathbf{H}})$, where problem $\mathcal{P}_{3}(\boldsymbol{\rho}, \boldsymbol{v}, \hat{\mathbf{H}})$ is the sample average approximation of problem $\mathcal{P}_{2}(\boldsymbol{\rho}, \boldsymbol{v}, \hat{\mathbf{H}})$ with $N$ samples. As stated in [9], problem $\mathcal{P}_{3}(\boldsymbol{\rho}, \boldsymbol{v}, \hat{\mathbf{H}})$ is equivalent to problem $\mathcal{P}_{2}(\boldsymbol{\rho}, \boldsymbol{v}, \hat{\mathbf{H}})$ w.p. 1 when $N$ approaches to infinity, due to the classical law of large number for random functions. That is to say, as $N \rightarrow \infty$, any stationary point of $\mathcal{P}_{3}(\boldsymbol{\rho}, \boldsymbol{v}, \hat{\mathbf{H}})$ is also a stationary point of problem $\mathcal{P}_{2}(\boldsymbol{\rho}, \boldsymbol{v}, \hat{\mathbf{H}})$ w.r.1. When $N$ is finite, Algorithm 2 converges to approximate stationary points of problem $\mathcal{P}_{2}(\boldsymbol{\rho}, \boldsymbol{v}, \hat{\mathbf{H}})$ with the exponential convergence rate $\hat{a}(\epsilon) e^{-N \hat{b}(\epsilon)}$. This is consequence of [14], Theorem 3.1, which provides a general convergence result for the original problem that satisfies the following assumptions: (a) The feasible set of optimization variables is a nonempty closed convex set; (b) The objective function of the original problem is continuously differentiable on the feasible set for any given random system states, and its gradient is Lipchitz continuous. Clearly, problem $\mathcal{P}_{2}(\boldsymbol{\rho}, \boldsymbol{v}, \hat{\mathbf{H}})$ satisfies the aforementioned assumption (a) and (b). This completes the proof.

Based on Theorem 2, the convergence of the proposed MOSSCA algorithm is summarized in the following theorem.

Theorem 3 (Convergence of the Algorithm 1). Given problem (2), suppose that $\tau>0$ in (9) and the step-sizes $\left\{\alpha_{t}\right\}$ and $\left\{\beta_{t}\right\}$ are chosen so that 
1) $\alpha_{t} \rightarrow 0, \frac{1}{\alpha_{t}} \leq O\left(t^{\kappa}\right)$ for some $\kappa \in(0,1), \sum_{t}\left(\alpha_{t}\right)^{2}<$ $\infty$

2) $\beta_{t} \rightarrow 0, \sum_{t} \beta_{t}=\infty, \sum_{t}\left(\beta_{t}\right)^{2}<\infty$,

3) $\lim _{t \rightarrow \infty} \beta_{t} / \alpha_{t}=0$.

Let $\left\{\boldsymbol{\rho}^{t}, \boldsymbol{v}^{t}, \mathbf{F}^{N}(i), \forall i \in \mathcal{T}_{t}\right\}_{t=1}^{\infty}$ denote the sequence of iterates generated by Algorithm 1, where $\mathbf{F}^{N}(i) \triangleq$ $\mathbf{F}^{N}\left(\boldsymbol{\rho}^{t}, \boldsymbol{v}^{t}, \hat{\mathbf{H}}(i)\right), i \in \mathcal{T}_{t}$. Then every limit point $\boldsymbol{v}^{*}, \boldsymbol{\rho}^{*}$ of $\left\{\boldsymbol{v}^{t}, \boldsymbol{\rho}^{t}\right\}_{t=1}^{\infty}$ almost surely satisfies

$$
\begin{gathered}
\boldsymbol{v}^{*}=\nabla_{\overline{\boldsymbol{\eta}}} g\left(\overline{\boldsymbol{\eta}}^{*}\right), \\
\left(\boldsymbol{\rho}-\boldsymbol{\rho}^{*}\right)^{T} \nabla_{\boldsymbol{\rho}}^{T} g\left(\overline{\boldsymbol{\eta}}\left(\boldsymbol{\rho}^{*}, \boldsymbol{\Theta}^{N}\left(\boldsymbol{v}^{*}, \boldsymbol{\rho}^{*}\right)\right)\right) \leq 0, \forall \boldsymbol{\rho} \in \mathbf{\Phi},
\end{gathered}
$$

where $\boldsymbol{\Theta}^{N}\left(\boldsymbol{v}^{*}, \boldsymbol{\rho}^{*}\right) \triangleq\left\{\mathbf{F}^{N}\left(\boldsymbol{v}^{*}, \boldsymbol{\rho}^{*}, \hat{\mathbf{H}}\right), \forall \hat{\mathbf{H}}\right\}$, and $\overline{\boldsymbol{\eta}}^{*} \triangleq$ $\overline{\boldsymbol{\eta}}\left(\boldsymbol{\rho}^{*}, \boldsymbol{\Theta}^{N}\left(\boldsymbol{v}^{*}, \boldsymbol{\rho}^{*}\right)\right)$. Moreover, $\forall \mathbf{F} \in \Lambda$, it satisfies

$$
\left(\mathbf{F}-\mathbf{F}^{N}(i)\right)^{T} J_{\mathbf{F}}\left(\boldsymbol{\rho}^{*}, \mathbf{F}^{N}(i) \mid \hat{\boldsymbol{H}}(i)\right) \nabla \overline{\bar{\eta}} g\left(\overline{\boldsymbol{\eta}}^{*}\right) \leq e(N),
$$

where $J_{\mathbf{F}}\left(\boldsymbol{\rho}^{*}, \mathbf{F}^{N}(i) \mid \hat{\boldsymbol{H}}(i)\right)$ is the Jacobian matrix of the vector $\overline{\boldsymbol{\eta}}^{\circ} \triangleq\left[\bar{\eta}_{1}^{\circ}, \cdots, \bar{\eta}_{K}^{\circ}\right]^{T}$ w.r.t. $\mathbf{F}$ at $\boldsymbol{\rho}=\boldsymbol{\rho}^{*}$ and $\mathbf{F}=\mathbf{F}^{N}(i)$, and $e(N)$ satisfies $\lim _{N \rightarrow \infty} e(N)=0$ almost surely.

Proof: Based on Theorem 2, Theorem 3 can be proven by a similar approach in [7]. Thus, we omit the details due to the limited space.

According to equation (15) in Theorem 3, it implies that the short-term solution $\mathbf{F}^{N}(i)$ found by Algorithm 2 must satisfy the stationary condition approximately with certain error $e(N)$ that converges to zero exponentially as $N \rightarrow \infty$. Moreover, the limiting point $\left(\boldsymbol{v}^{*}, \boldsymbol{\rho}^{*}\right)$ generated by Algorithm 1 also satisfies the stationary conditions in (13) and (14), respectively. Thus, Algorithm 1 converges to stationary solutions of the mixedtimescale optimization problem $\mathcal{P}$. Note that since $e(N)$ converges to zero exponentially, Algorithm 2 with a small $N$ can already achieve a good performance and avoids excessive computational complexity.

\section{Simulation Results and Discussions}

We consider a single-cell of radius $100 \mathrm{~m}$, where BS is equipped with 64 antennas. There are 12 devices randomly distributed in the cell. We adopt a geometric channel model with a half-wavelength space ULA for simulations [7]. The channel between BS and device $k$ is given by $\boldsymbol{h}_{k}=\sum_{i=1}^{N_{p}} \varepsilon_{k, i} \mathrm{a}\left(\varphi_{k, i}\right)$, where $\mathrm{a}(\varphi)$ is the array response vector, $\varphi_{k, i}$ 's are Laplacian distributed with an angular spread $\sigma_{\mathrm{AS}}=10, \varepsilon_{k, i} \sim \mathcal{C N}\left(0, \sigma_{k, i}^{2}\right), \sigma_{k, i}^{2}$ are randomly generated from an exponential distribution and normalized such that $\sum_{i=1}^{N_{p}} \sigma_{k, i}^{2}=G_{n, k}, G_{k}$ is the average channel gain determined by the pathloss model $30.6+36.7 \log 10\left(\mathrm{ds}_{k}\right)$ [15], and $\mathrm{ds}_{k}$ is the distance between BS and device $k$ in meters. We consider $N_{p}=6$ channel paths for each device. The transmit power budget for BS is $P_{\max }=10 \mathrm{dBm}$. We set $N=200, S_{k}=24$ $\mathrm{mW}, a_{k}=150, b_{k}=0.014, \gamma_{k}=10, \omega_{k}^{2}=-40 \mathrm{~dB}$, $\sigma_{k}^{2}=-60 \mathrm{dBm}$ and $\delta_{k}^{2}=-50 \mathrm{dBm}$. There are $T_{s}=10$ time slots in each frame and the slot size is $2 \mathrm{~ms}$. The coherence interval $T=400$, which corresponds to a coherence time of $2 \mathrm{~ms}$ and a coherence bandwidth of $200 \mathrm{kHz}$ [16]. The coherence time for the channel statistics is assumed to be 10

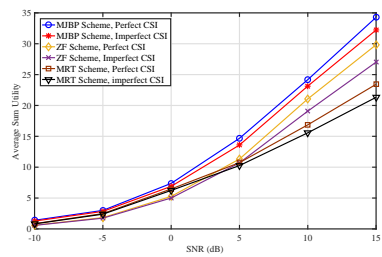

(a)

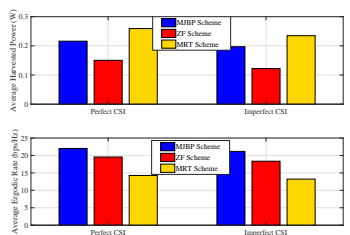

(b)
Fig. 3: (a) Utility performance versus SNR. (b) Tradeoff comparison for different schemes $(M=64, K=12$, and $\mathrm{SNR}=10 \mathrm{~dB}$ ).

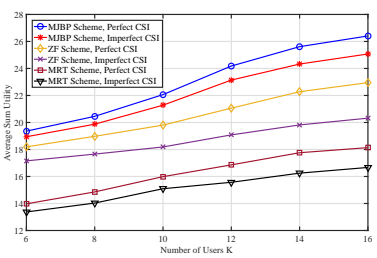

(a)

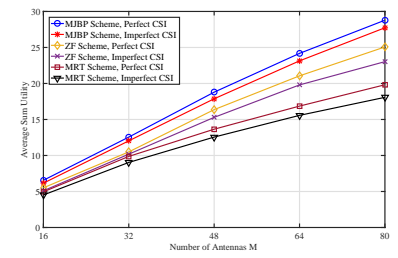

(b)
Fig. 4: (a) Utility performance versus the number of devices $K$. (b) Utility performance versus the number of antennas $M$.

s. We use the average sum utility $g(\hat{\boldsymbol{\eta}})=\sum_{k=1}^{K} \hat{\eta}_{k}$ as an example to illustrate the advantages of the proposed scheme. Two schemes are included as baselines: 1) maximum ratio transmission (MRT) scheme, which is obtained by fixing the MRT beamformer [3]; 2) zero-forcing (ZF) scheme, which is obtained by fixing the ZF beamformer [5]. The power splitters of both MRT and ZF scheme are obtained by the long-term optimization.

In Fig 3(a), we plot the utility performance versus the signalto-noise ratio (SNR). We can see that as the SNR increases, the average sum utility of all schemes increases gradually. It is observed that the average sum utility achieved by the proposed MJBP scheme is higher than that achieved by the other schemes for moderate and large SNR. This indicates that the proposed MJBP scheme can better mitigate the multidevice interference to achieve better tradeoff between the average ergodic rate and the average harvested power, which is further validated in Fig 3(b).

In Fig 4(a), we plot the utility performance versus the number of devices $K$. We observe that the proposed MJBP scheme achieves significant gain over MRT scheme and ZF scheme, which demonstrates the importance of mixed-timescale joint optimization. Moreover, as the number of devices $K$ increases, the performance gap between the proposed MJBP scheme and other competing schemes becomes larger.

In Fig 4(b), we plot the utility performance versus the number of antennas at BS. It shows that the performance of all these schemes is monotonically increasing with the number of antennas. Again, it is seen that the proposed MJBP scheme outperforms all the other schemes for all $M$ regime.

\section{CONClusion}

In this letter, we considered mixed-timescale joint beamforming and power splitting (MJBP) scheme in the downlink 
transmission of massive MIMO aided SWIPT IoT network to maximize the network utility under the power budget constraint. We proposed a MO-SSCA algorithm to find stationary solutions of the mixed-timescale non-convex stochastic optimization problem. Simulations verify that the proposed MJBP scheme achieves significant gain over existing schemes.

\section{REFERENCES}

[1] M. Swan, "Sensor mania! the Internet of things, wearable computing, objective metrics, and the quantified self 2.0," J. Sens. Actuator Netw, vol. 1, no. 3, pp. 217-253, 2012.

[2] R. Zhang and C. K. HO, "MIMO broadcasting for simultaneous wireless information and power transfer," IEEE Trans. Wireless Commun. vol. 12, no. 5, pp. 1989-2001, May 2013.

[3] Q. Shi, L. Liu, W. Xu, and R. Zhang, "Joint transmit beamforming and receive power splitting for MISO SWIPT systems," IEEE Trans. Wireless Commun., vol. 13, no. 6, pp. 3269-3280, Apr 2014.

[4] Q. Shi, W. Xu, T. Chang, Y. Wang, and E. Song, "Joint beamforming and power splitting for MISO interference channel with SWIPT: An SOCP relaxation and decentralized algorithm," IEEE Trans. Signal Process., vol. 62, no. 23, pp. 6194-6208, Dec 2014.

[5] G. Dong, H. Zhang, and D. Yuan, "Optimal downlink transmission in Massive MIMO enabled SWIPT systems with zero-forcing precoding," IEEE Global Commun. Conf., Dec 2014.

[6] G. Caire, "On the ergodic rate lower bounds with applications to masssive MIMO," IEEE Trans. Wireless Commun., vol. 17, no. 5, pp. 3258-3268, May 2018.

[7] A. Liu, X. Chen, W. Yu, V. Lau, and M. Zhao, "Two-timescale hybrid compression and forward for massive MIMO aided C-RAN," IEEE Trans. Signal Process., vol. 67, no. 9, pp. 2484-2498, Mar 2019.

[8] E. Boshkovska, D. Ng, N. Zlatanov, and R. Schober, "Practical nonlinear energy harvesting model and resource allocation for SWIPT systems," IEEE Commun. Lett., vol. 19, no. 12, pp. 2082-2085, Dec. 2015.

[9] A. Shapiro, D. Dentcheva, and A. Ruszczynski, Lecture on stochastic programming: modeling and theory. SIAM, 2009.

[10] K. Shen and W. Yu, "Fractional programming for communication systems - Part II: Uplink scheduling via matching," IEEE Trans. Signal Process., vol. 66, no. 10, pp. 2631-2644, Mar 2018.

[11] M. W. Jacobson and J. A. Fessler, "An expanded theoretical treatment of iteration-dependent majorize-minimize algorithms," IEEE Transactions on Image Processing, vol. 16, no. 10, pp. 2411-2422, Oct 2007.

[12] "CVX Research, Inc. CVX: Matlab software for disciplined convex programming, version 2.0 beta." Sep, 2012. [Online]. Available: http:// cvxr.com/cvx.

[13] K. Shen, W. Yu, L. Zhao, and D. P. Palomar, "Optimal of MIMO deviceto-device networks via matrix fractional programming: A minorizationmaximization approach," arXiv.org:1808.05678, 2019.

[14] H. Sun and H. Xu, "A note on uniform exponential convergence of sample average approximation of random functions," J. Math. Anal. Appl, pp. 698-708, 2012.

[15] Technical Specification Group Radio Access Network; Further Advancements for E-UTRA Physical Layer Aspects, 3GPP TR 36.814. [Online]. Available: http://www.3gpp.org

[16] T. Marzetta, E. G. Larsson, H. Yang, and H. Ngo, Fundamentals of Massive MIMO. Cambridge, U.K.: Cambridge University Press, 2016. 